Mathematical Modelling and Analysis

Volume 21 Number 4, July 2016, 533-549

http://dx.doi.org/10.3846/13926292.2016.1186119

(c) Vilnius Gediminas Technical University, 2016
Publisher: Taylor\&Francis and VGTU

http://www.tandfonline.com/TMMA

ISSN: $1392-6292$

eISSN: 1648-3510

\title{
Gradient Based Iterative Algorithm to Solve General Coupled Discrete-Time Periodic Matrix Equations over Generalized Reflexive Matrices
}

\section{Masoud Hajarian}

Department of Mathematics, Faculty of Mathematical Sciences, Shahid Beheshti University

General Campus, Evin, 19839 Tehran, Iran

E-mail: m_hajarian@sbu.ac.ir; mhajarian@aut.ac.ir

Received December 13, 2015; revised April 27, 2016; published online July 1, 2016

\begin{abstract}
The discrete-time periodic matrix equations are encountered in periodic state feedback problems and model reduction of periodic descriptor systems. The aim of this paper is to compute the generalized reflexive solutions of the general coupled discrete-time periodic matrix equations. We introduce a gradient-based iterative (GI) algorithm for finding the generalized reflexive solutions of the general coupled discretetime periodic matrix equations. It is shown that the introduced GI algorithm always converges to the generalized reflexive solutions for any initial generalized reflexive matrices. Finally, two numerical examples are investigated to confirm the efficiency of GI algorithm.
\end{abstract}

Keywords: discrete-time periodic matrix equation, iterative algorithm, gradient based iterative algorithm, generalized reflexive solution .

AMS Subject Classification: 15A24; 39B42; 65F10; 65F30.

\section{Introduction}

Let us begin with some notations and definitions. The symbols $A^{T}, \operatorname{tr}(A)$ and $\|A\|$ will stand for the transpose, the trace and the Frobenius norm of a matrix $A \in \mathbb{R}^{m \times n}$, respectively. For a matrix $A \in \mathbb{R}^{m \times n}$, the so-called stretching function $\operatorname{vec}(A)$ is defined by $\operatorname{vec}(A)=\left(a_{1}^{T}, a_{2}^{T}, \ldots, a_{n}^{T}\right)^{T}$, where $a_{k}$ is the $k$-th column of $A$. The notation $A \otimes B$ represents the Kronecker product of matrices $A$ and $B$. A matrix $P \in \mathbb{R}^{n \times n}$ is called a generalized reflection matrix if $P=P^{T}$ and $P^{2}=I$. Throughout, we always suppose that $P, Q \in \mathbb{R}^{n \times n}$ are given generalized reflection matrices. If $A=P A Q$ then $A \in \mathbb{R}^{n \times n}$ is called a generalized reflexive matrix with respect to $(P, Q)[5]$. The symbol $\mathbb{R}_{r}^{n \times n}(P, Q)$ denotes the set of $n \times n$ generalized reflexive matrices with respect to $(P, Q)$. Obviously every matrix $A \in \mathbb{R}^{n \times n}$ is also a generalized reflexive matrix with respect to $(I, I)$. In [5], three important applications of 
the generalized reflexive matrices were proposed.

The linear systems and linear matrix equations have several applications in several problems of applied mathematics and engineering [6, 26, 29,32,33]. For example, the stability of discrete-time linear periodic system

$$
x(k+1)=A_{k} x(k)+B_{k} u(k), \quad \forall k \in \mathbb{Z}
$$

is closely related with the following discrete-time periodic Lyapunov matrix equations $[3,35]$

$$
A_{k} P_{k} A_{k}^{T}-P_{k+1}=-B_{k} B_{k}^{T}, \quad \forall k \in \mathbb{Z}
$$

and

$$
A_{k}^{T} P_{k+1} A_{k}-P_{k}=-Q_{k}, \quad \forall k \in \mathbb{Z} .
$$

In the model reduction and stability analysis of the linear periodic time-varying descriptor systems

$$
E_{i} x_{i+1}=A_{i} x_{i}+B_{i} u_{i}, \quad y_{i}=C_{i} x_{i}, \quad \forall i \in \mathbb{Z}
$$

we need to solve the following generalized projected periodic discrete-time algebraic Lyapunov matrix equations $[2,6,27]$

$$
\left\{\begin{array}{l}
A_{i} X_{i} A_{i}^{T}-E_{i} X_{i+1} E_{i}^{T}=Q_{l}(i) B_{i} B_{i}^{T} Q_{l}(i)^{T}, \\
X_{i}=Q_{r}(i) G_{i} Q_{r}(i)^{T} .
\end{array}\right.
$$

The applications of linear matrix equations have motivated both mathematicians and engineers to construct methods catering to solve linear matrix equations $[1,4,6,7,8,9,19,23,25]$. Based on Smith iterations [24], iterative methods were developed for periodic standard Lyapunov matrix equations and projected generalized Lyapunov matrix equations $[27,28]$. Kressner introduced new variants of the squared Smith iteration and Krylov subspace based methods for the approximate solution of discrete-time periodic Lyapunov matrix equations [20]. In [17], Granat et al. presented novel recursive blocked algorithms for solving various periodic triangular matrix equations. In this paper, we propose a GI algorithm to find the generalized reflexive solutions of the general coupled discrete-time periodic matrix equations

$$
\left\{\begin{array}{l}
A_{1, i} X_{i} B_{1, i}+C_{1, i} X_{i+1} D_{1, i}=E_{1, i} \\
A_{2, i} X_{i} B_{2, i}+C_{2, i} X_{i+1} D_{2, i}=E_{2, i}
\end{array}\right.
$$

for $i=1,2, \ldots$, where the coefficient matrices $A_{1, i}, C_{1, i} \in \mathbb{R}^{p_{1} \times n}, A_{2, i}, C_{2, i} \in$ $\mathbb{R}^{p_{2} \times n}, B_{1, i}, D_{1, i} \in \mathbb{R}^{n \times q_{1}}, B_{2, i}, D_{2, i} \in \mathbb{R}^{n \times q_{2}}, E_{1, i} \in \mathbb{R}^{p_{1} \times q_{1}}, E_{2, i} \in \mathbb{R}^{p_{2} \times q_{2}}$ and the generalized reflexive solutions $X_{i} \in \mathbb{R}_{r}^{n \times n}(P, Q)$ are periodic with period $\theta$, i.e., $A_{1, i+\theta}=A_{1, i}, A_{2, i+\theta}=A_{2, i}, C_{1, i+\theta}=C_{1, i}, C_{2, i+\theta}=C_{2, i}, D_{1, i+\theta}=D_{1, i}$, $D_{2, i+\theta}=D_{2, i}, E_{1, i+\theta}=E_{1, i}, E_{2, i+\theta}=E_{2, i}$ and $X_{i+\theta}=X_{i}$. It is worth mentioning that the generalized reflexive solutions of the general coupled discretetime periodic matrix equations (1.4) have not been dealt with yet. Meanwhile the general coupled discrete-time periodic matrix equations (1.4) contain various linear discrete-time periodic matrix equations as special cases such as (1.1), 
(1.2) and (1.3).

The remaining parts of this paper are organized as follows. In Section 2, first a GI algorithm is proposed for solving (1.4) over the generalized reflexive matrices. Then by analysis of convergence we prove that the proposed algorithm consistently converges to the generalized reflexive solutions for any initial generalized reflexive matrices. Theoretical results are verified on the relevant numerical examples in Section 3. Section 4 ends this paper with a brief conclusion.

\section{Main results}

In this section, first we obtain the conditions for solvability of (1.4) over the generalized reflexive matrices. Then a GI algorithm and its convergence analysis are given.

It is easily shown that the general coupled discrete-time periodic matrix equations (1.4) over the generalized reflexive matrixes are equivalent to the following general coupled matrix equations

$$
\left\{\begin{array}{l}
\mathcal{A}_{1} \mathcal{X} \mathcal{B}_{1}+\mathcal{C}_{1} \mathcal{X} \mathcal{D}_{1}=\mathcal{E}_{1} \\
\mathcal{A}_{2} \mathcal{X} \mathcal{B}_{2}+\mathcal{C}_{2} \mathcal{X} \mathcal{D}_{2}=\mathcal{E}_{2} \\
\mathcal{A}_{1} \mathcal{P} \mathcal{X} \mathcal{Q B}_{1}+\mathcal{C}_{1} \mathcal{P} \mathcal{X} \mathcal{D}_{1}=\mathcal{E}_{1} \\
\mathcal{A}_{2} \mathcal{P} \mathcal{X} \mathcal{Q B}_{2}+\mathcal{C}_{2} \mathcal{P} \mathcal{X} \mathcal{Q} \mathcal{D}_{2}=\mathcal{E}_{2}
\end{array}\right.
$$

where

$$
\begin{gathered}
\mathcal{A}_{j}=\left(\begin{array}{cccc}
0 & \ldots & 0 & A_{j, 1} \\
A_{j, 2} & & & 0 \\
& \ddots & & \vdots \\
0 & & A_{j, \theta} & 0
\end{array}\right), \quad \mathcal{B}_{j}=\left(\begin{array}{cccc}
0 & B_{j, 2} & & 0 \\
\vdots & & \ddots & \\
0 & & & B_{j, \theta} \\
B_{j, 1} & 0 & \ldots & 0
\end{array}\right), \\
\mathcal{C}_{j}=\operatorname{diag}\left(C_{j, 1}, C_{j, 2}, \ldots, C_{j, \theta}\right), \mathcal{D}_{j}=\operatorname{diag}\left(D_{j, 1}, D_{j, 2}, \ldots, D_{j, \theta}\right) \\
\mathcal{E}_{j}=\operatorname{diag}\left(E_{j, 1}, E_{j, 2}, \ldots, E_{j, \theta}\right), \quad \mathcal{X}=\operatorname{diag}\left(X_{2}, X_{3}, \ldots, X_{\theta}, X_{1}\right) \\
\mathcal{P}=\operatorname{diag}(P, P, \ldots, P), \quad \mathcal{Q}=\operatorname{diag}(Q, Q, \ldots, Q)
\end{gathered}
$$

for $j=1,2$. By using Kronecker product and vectorization operator, the general coupled matrix equations (2.1) can be transformed into the linear system $A x=b$ with the following parameters:

$$
A=\left(\begin{array}{c}
\mathcal{B}_{1}^{T} \otimes \mathcal{A}_{1}+\mathcal{D}_{1}^{T} \otimes \mathcal{C}_{1} \\
\mathcal{B}_{2}^{T} \otimes \mathcal{A}_{2}+\mathcal{D}_{2}^{T} \otimes \mathcal{C}_{2} \\
\mathcal{B}_{1}^{T} \mathcal{Q} \otimes \mathcal{A}_{1} \mathcal{P}+\mathcal{D}_{1}^{T} \mathcal{Q} \otimes \mathcal{C}_{1} \mathcal{P} \\
\mathcal{B}_{2}^{T} \mathcal{Q} \otimes \mathcal{A}_{2} \mathcal{P}+\mathcal{D}_{2}^{T} \mathcal{Q} \otimes \mathcal{C}_{2} \mathcal{P}
\end{array}\right), \quad x=\operatorname{vec}(\mathcal{X}), \quad b=\left(\begin{array}{r}
\operatorname{vec}\left(\mathcal{E}_{1}\right) \\
\operatorname{vec}\left(\mathcal{E}_{2}\right) \\
\operatorname{vec}\left(\mathcal{E}_{1}\right) \\
\operatorname{vec}\left(\mathcal{E}_{2}\right)
\end{array}\right)
$$

By applying (2.2), we can present the following lemma.

Lemma 1. The general coupled discrete-time periodic matrix equations (1.4) have a unique generalized reflexive solution group $\left(X_{1}, X_{2}, \ldots, X_{\theta}\right)$ if and only 
if $\operatorname{rank}((A, b))=\operatorname{rank}(A)$ and $A$ has a full column rank; in this case, the homogenous general coupled discrete-time periodic matrix equations

$$
\left\{\begin{array}{l}
A_{1, i} X_{i} B_{1, i}+C_{1, i} X_{i+1} D_{1, i}=0, \\
A_{2, i} X_{i} B_{2, i}+C_{2, i} X_{i+1} D_{2, i}=0,
\end{array} \quad i=1,2, \ldots\right.
$$

have a unique generalized reflexive solution group $\left(X_{1}, X_{2}, \ldots, X_{\theta}\right)=0$.

Obviously the size of the coefficient matrices of the general coupled matrix equations (2.1) and the linear system (2.2) is large. When the size of coefficient matrices is large, the iterative methods such as $[10,18,22]$ will consume more computer time and memory space. Also in this case, the obtained solutions are not accurate enough. To overcome the complications, we directly extend the GI algorithm to solve (1.4) over the generalized reflexive matrices. One of the famous method for solving the linear system $A x=b$ is the GI algorithm $[12,13,14]$ as follows:

$$
x^{(k+1)}=x^{(k)}+\delta A^{T}\left(b-A x^{(k)}\right), \quad 0<\delta<\frac{2}{\|A\|^{2}} .
$$

In recent years the GI algorithms have gained much attention for solving linear matrix equations $[14,15,16]$. In [11,12,13], Ding and Chen proposed the GI algorithms for solving matrix equations. Zhou et al. constructed a GI algorithm to approximate the solutions to the coupled linear matrix equations [34]. By defining a relaxation parameter, Niu et al. proposed a relaxed GI algorithm for solving Sylvester matrix equations [21]. Different from the GI algorithm presented in [11] and the relaxed GI algorithm given in [21], Wang et al. introduced a modified GI algorithm for solving Sylvester matrix equations [30]. In [31], Wang and Liao obtained the optimal convergence factor of the GI algorithm for linear matrix equations. Based on the (2.1), (2.2) and (2.3), we present the following GI algorithm for solving (1.4) over the generalized reflexive matrices:

\section{Algorithm 1. (GI algorithm to solve (1.4) over the generalized reflexive matrices)}

Step 1 Choose the initial generalized reflexive matrices $X_{i}(1) \in \mathbb{R}_{r}^{n \times n}(P, Q)$ for $i=1,2, \ldots, \theta$ and a parameter $\delta>0$;

Step 2 Set $X_{\theta+1}(1)=X_{1}(1), X_{0}(1)=X_{\theta}(1), C_{j, 0}=C_{j, \theta}$ and $D_{j, 0}=D_{j, \theta}$ for $j=1,2$;

\section{Step 3 Compute}

$$
R_{j, i}(1)=E_{j, i}-A_{j, i} X_{i}(1) B_{j, i}-C_{j, i} X_{i+1}(1) D_{j, i}, \quad i=1,2, \ldots, \theta, \quad j=1,2,
$$

and set $R_{j, 0}(1)=R_{j, \theta}(1)$ for $j=1,2$;

Step 4 For $k=1,2, \ldots$, compute

$$
X_{i}(k+1)=X_{i}(k)+\frac{\delta}{2}\left[A_{1, i}^{T} R_{1, i}(k) B_{1, i}^{T}+A_{2, i}^{T} R_{2, i}(k) B_{2, i}^{T}+C_{1, i-1}^{T} R_{1, i-1}(k) D_{1, i-1}^{T}\right.
$$




$$
\begin{gathered}
+C_{2, i-1}^{T} R_{2, i-1}(k) D_{2, i-1}^{T}+P A_{1, i}^{T} R_{1, i}(k) B_{1, i}^{T} Q+P A_{2, i}^{T} R_{2, i}(k) B_{2, i}^{T} Q \\
\left.+P C_{1, i-1}^{T} R_{1, i-1}(k) D_{1, i-1}^{T} Q+P C_{2, i-1}^{T} R_{2, i-1}(k) D_{2, i-1}^{T} Q\right], \quad i=1,2, \ldots, \theta, \\
X_{\theta+1}(k+1)=X_{1}(k+1), \quad X_{0}(k+1)=X_{\theta}(k+1), \\
R_{j, i}(k+1)=E_{j, i}-A_{j, i} X_{i}(k+1) B_{j, i}-C_{j, i} X_{i+1}(k+1) D_{j, i}, i=1,2, \ldots, \theta, \quad j=1,2, \\
R_{j, 0}(k+1)=R_{j, \theta}(k+1), \quad j=1,2 .
\end{gathered}
$$

Stopping criterion. To check convergence, we use the stopping criterion

$$
\sqrt{\sum_{i=1}^{\theta}\left(\left\|R_{1, i}(k)\right\|^{2}+\left\|R_{2, i}(k)\right\|^{2}\right)} \leq \text { tol }
$$

where tol is a chosen fixed threshold.

Remark 1 . From the above algorithm, we can easily see that $X_{i}(k) \in \mathbb{R}_{r}^{n \times n}(P, Q)$ for $i=1,2, \ldots, \theta$.

In the following theorem, we proceed to prove the convergence of Algorithm 1 to the generalized reflexive solutions of (1.4).

Theorem 1. Suppose that the general coupled discrete-time periodic matrix equations (1.4) have a unique generalized reflexive solution group $\left(X_{1}^{*}, X_{2}^{*}\right.$, $\left.\ldots, X_{\theta}^{*}\right)$. If the parameter $\delta$ satisfies the inequality

$$
0<\delta<\frac{2}{\sum_{i=1}^{\theta}\left(\left\|A_{1, i} B_{1, i}\right\|^{2}+\left\|C_{1, i} D_{1, i}\right\|^{2}+\left\|A_{2, i} B_{2, i}\right\|^{2}+\left\|C_{2, i} D_{2, i}\right\|^{2}\right)}
$$

then for any initial generalized reflexive matrix group $\left(X_{1}(1), X_{2}(1), \ldots, X_{\theta}(1)\right)$, the iterative solution group $\left(X_{1}(k), X_{2}(k), \ldots, X_{\theta}(k)\right)$ generated by Algorithm 1 converges to the generalized reflexive group $\left(X_{1}^{*}, X_{2}^{*}, \ldots, X_{\theta}^{*}\right)$, that is

$$
\lim _{k \rightarrow \infty} X_{i}(k)=X_{i}^{*}, \quad \text { for } \quad i=1,2, \ldots, \theta .
$$

Proof. To prove this theorem, first we define the error matrices in the $k$-th iteration of Algorithm 1 as

$$
\tilde{X}_{i}(k)=X_{i}(k)-X_{i}^{*}, \quad \text { for } \quad i=1,2, \ldots, \theta .
$$

By using the error matrices, we can obtain the residual matrices in the $k$-th iteration as the following form

$$
R_{j, i}(k)=-A_{j, i} \tilde{X}_{i}(k) B_{j, i}-C_{j, i} \tilde{X}_{i+1}(k) D_{j, i}, \quad \text { for } i=1,2, \ldots, \theta, \quad j=1,2 .
$$

This implies that

$$
\widetilde{X}_{i}(k+1)=\widetilde{X}_{i}(k)-\frac{\delta}{2}\left[A_{1, i}^{T}\left(A_{1, i} \widetilde{X}_{i}(k) B_{1, i}+C_{1, i} \widetilde{X}_{i+1}(k) D_{1, i}\right) B_{1, i}^{T}\right.
$$




$$
\begin{gathered}
+A_{2, i}^{T}\left(A_{2, i} \widetilde{X}_{i}(k) B_{2, i}+C_{2, i} \widetilde{X}_{i+1}(k) D_{2, i}\right) B_{2, i}^{T} \\
+C_{1, i-1}^{T}\left(A_{1, i-1} \widetilde{X}_{i-1}(k) B_{1, i-1}+C_{1, i-1} \widetilde{X}_{i}(k) D_{1, i-1}\right) D_{1, i-1}^{T} \\
+C_{2, i-1}^{T}\left(A_{2, i-1} \widetilde{X}_{i-1}(k) B_{2, i-1}+C_{2, i-1} \widetilde{X}_{i}(k) D_{2, i-1}\right) D_{2, i-1}^{T} \\
+P A_{1, i}^{T}\left(A_{1, i} \widetilde{X}_{i}(k) B_{1, i}+C_{1, i} \widetilde{X}_{i+1}(k) D_{1, i}\right) B_{1, i}^{T} Q \\
+P A_{2, i}^{T}\left(A_{2, i} \widetilde{X}_{i}(k) B_{2, i}+C_{2, i} \widetilde{X}_{i+1}(k) D_{2, i}\right) B_{2, i}^{T} Q \\
+P C_{1, i-1}^{T}\left(A_{1, i-1} \widetilde{X}_{i-1}(k) B_{1, i-1}+C_{1, i-1} \widetilde{X}_{i}(k) D_{1, i-1}\right) D_{1, i-1}^{T} Q \\
\left.+P C_{2, i-1}^{T}\left(A_{2, i-1} \widetilde{X}_{i-1}(k) B_{2, i-1}+C_{2, i-1} \widetilde{X}_{i}(k) D_{2, i-1}\right) D_{2, i-1}^{T} Q\right] .
\end{gathered}
$$

For $i=1,2, \ldots, \theta$, by applying (2.5) we can obtain

$$
\begin{gathered}
\left\|\widetilde{X}_{i}(k+1)\right\|^{2}=\operatorname{tr}\left(\widetilde{X}_{i}(k+1)^{T} \widetilde{X}_{i}(k+1)\right) \\
=\left\|\widetilde{X}_{i}(k)\right\|^{2}-\delta \operatorname{tr}\left(\widetilde{X}_{i}(k)^{T} A_{1, i}^{T}\left(A_{1, i} \widetilde{X}_{i}(k) B_{1, i}+C_{1, i} \widetilde{X}_{i+1}(k) D_{1, i}\right) B_{1, i}^{T}\right. \\
+\widetilde{X}_{i}(k)^{T} A_{2, i}^{T}\left(A_{2, i} \widetilde{X}_{i}(k) B_{2, i}+C_{2, i} \widetilde{X}_{i+1}(k) D_{2, i}\right) B_{2, i}^{T} \\
+\widetilde{X}_{i}(k)^{T} C_{1, i-1}^{T}\left(A_{1, i-1} \widetilde{X}_{i-1}(k) B_{1, i-1}+C_{1, i-1} \widetilde{X}_{i}(k) D_{1, i-1}\right) D_{1, i-1}^{T} \\
+\widetilde{X}_{i}(k)^{T} C_{2, i-1}^{T}\left(A_{2, i-1} \widetilde{X}_{i-1}(k) B_{2, i-1}+C_{2, i-1} \widetilde{X}_{i}(k) D_{2, i-1}\right) D_{2, i-1}^{T} \\
+\widetilde{X}_{i}(k)^{T} P A_{1, i}^{T}\left(A_{1, i} \widetilde{X}_{i}(k) B_{1, i}+C_{1, i} \widetilde{X}_{i+1}(k) D_{1, i}\right) B_{1, i}^{T} Q \\
+\widetilde{X}_{i}(k)^{T} P A_{2, i}^{T}\left(A_{2, i} \widetilde{X}_{i}(k) B_{2, i}+C_{2, i} \widetilde{X}_{i+1}(k) D_{2, i}\right) B_{2, i}^{T} Q \\
+\widetilde{X}_{i}(k)^{T} P C_{1, i-1}^{T}\left(A_{1, i-1} \widetilde{X}_{i-1}(k) B_{1, i-1}+C_{1, i-1} \widetilde{X}_{i}(k) D_{1, i-1}\right) D_{1, i-1}^{T} Q \\
\left.+\widetilde{X}_{i}(k)^{T} P C_{2, i-1}^{T}\left(A_{2, i-1} \widetilde{X}_{i-1}(k) B_{2, i-1}+C_{2, i-1} \widetilde{X}_{i}(k) D_{2, i-1}\right) D_{2, i-1}^{T} Q\right) \\
+\frac{\delta^{2}}{4} \| A_{1, i}^{T}\left(A_{1, i} \widetilde{X}_{i}(k) B_{1, i}+C_{1, i} \widetilde{X}_{i+1}(k) D_{1, i}\right) B_{1, i}^{T} \\
+A_{2, i}^{T}\left(A_{2, i} \widetilde{X}_{i}(k) B_{2, i}+C_{2, i} \widetilde{X}_{i+1}(k) D_{2, i}\right) B_{2, i}^{T} \\
+C_{1, i-1}^{T}\left(A_{1, i-1} \widetilde{X}_{i-1}(k) B_{1, i-1}+C_{1, i-1} \widetilde{X}_{i}(k) D_{1, i-1}\right) D_{1, i-1}^{T} \\
+C_{2, i-1}^{T}\left(A_{2, i-1} \widetilde{X}_{i-1}(k) B_{2, i-1}+C_{2, i-1} \widetilde{X}_{i}(k) D_{2, i-1}\right) D_{2, i-1}^{T} \\
+P A_{1, i}^{T}\left(A_{1, i} \widetilde{X}_{i}(k) B_{1, i}+C_{1, i} \widetilde{X}_{i+1}(k) D_{1, i}\right) B_{1, i}^{T} Q \\
+P A_{2, i}^{T}\left(A_{2, i} \widetilde{X}_{i}(k) B_{2, i}+C_{2, i} \widetilde{X}_{i+1}(k) D_{2, i}\right) B_{2, i}^{T} Q
\end{gathered}
$$




$$
\begin{aligned}
& +P C_{1, i-1}^{T}\left(A_{1, i-1} \widetilde{X}_{i-1}(k) B_{1, i-1}+C_{1, i-1} \widetilde{X}_{i}(k) D_{1, i-1}\right) D_{1, i-1}^{T} Q \\
& +P C_{2, i-1}^{T}\left(A_{2, i-1} \widetilde{X}_{i-1}(k) B_{2, i-1}+C_{2, i-1} \widetilde{X}_{i}(k) D_{2, i-1}\right) D_{2, i-1}^{T} Q \|^{2} \\
& =\left\|\widetilde{X}_{i}(k)\right\|^{2}-2 \delta \operatorname{tr}\left(A_{1, i} \tilde{X}_{i}(k) B_{1, i}\left(A_{1, i} \tilde{X}_{i}(k) B_{1, i}+C_{1, i} \tilde{X}_{i+1}(k) D_{1, i}\right)^{T}\right. \\
& +A_{2, i} \tilde{X}_{i}(k) B_{2, i}\left(A_{2, i} \tilde{X}_{i}(k) B_{2, i}+C_{2, i} \tilde{X}_{i+1}(k) D_{2, i}\right)^{T} \\
& +C_{1, i-1} \widetilde{X}_{i}(k) D_{1, i-1}\left(A_{1, i-1} \widetilde{X}_{i-1}(k) B_{1, i-1}+C_{1, i-1} \widetilde{X}_{i}(k) D_{1, i-1}\right)^{T} \\
& \left.+C_{2, i-1} \widetilde{X}_{i}(k) D_{2, i-1}\left(A_{2, i-1} \widetilde{X}_{i-1}(k) B_{2, i-1}+C_{2, i-1} \widetilde{X}_{i}(k) D_{2, i-1}\right)^{T}\right) \\
& +\delta^{2} \| A_{1, i}^{T}\left(A_{1, i} \widetilde{X}_{i}(k) B_{1, i}+C_{1, i} \tilde{X}_{i+1}(k) D_{1, i}\right) B_{1, i}^{T} \\
& +C_{1, i-1}^{T}\left(A_{1, i-1} \widetilde{X}_{i-1}(k) B_{1, i-1}+C_{1, i-1} \widetilde{X}_{i}(k) D_{1, i-1}\right) D_{1, i-1}^{T} \\
& +A_{2, i}^{T}\left(A_{2, i} \tilde{X}_{i}(k) B_{2, i}+C_{2, i} \tilde{X}_{i+1}(k) D_{2, i}\right) B_{2, i}^{T} \\
& +C_{2, i-1}^{T}\left(A_{2, i-1} \widetilde{X}_{i-1}(k) B_{2, i-1}+C_{2, i-1} \tilde{X}_{i}(k) D_{2, i-1}\right) D_{2, i-1}^{T} \|^{2} \\
& =\left\|\widetilde{X}_{i}(k)\right\|^{2}-2 \delta \operatorname{tr}\left(A_{1, i} \widetilde{X}_{i}(k) B_{1, i}\left(A_{1, i} \widetilde{X}_{i}(k) B_{1, i}+C_{1, i} \widetilde{X}_{i+1}(k) D_{1, i}\right)^{T}\right. \\
& +A_{2, i} \widetilde{X}_{i}(k) B_{2, i}\left(A_{2, i} \widetilde{X}_{i}(k) B_{2, i}+C_{2, i} \widetilde{X}_{i+1}(k) D_{2, i}\right)^{T} \\
& +C_{1, i} \widetilde{X}_{i+1}(k) D_{1, i}\left(A_{1, i} \widetilde{X}_{i}(k) B_{1, i}+C_{1, i} \widetilde{X}_{i+1}(k) D_{1, i}\right)^{T} \\
& \left.+C_{2, i} \widetilde{X}_{i+1}(k) D_{2, i}\left(A_{2, i} \widetilde{X}_{i}(k) B_{2, i}+C_{2, i} \tilde{X}_{i+1}(k) D_{2, i}\right)^{T}\right) \\
& +\delta^{2} \| A_{1, i}^{T}\left(A_{1, i} \widetilde{X}_{i}(k) B_{1, i}+C_{1, i} \tilde{X}_{i+1}(k) D_{1, i}\right) B_{1, i}^{T} \\
& +C_{1, i}^{T}\left(A_{1, i} \widetilde{X}_{i}(k) B_{1, i}+C_{1, i} \widetilde{X}_{i+1}(k) D_{1, i}\right) D_{1, i}^{T} \\
& +A_{2, i}^{T}\left(A_{2, i} \tilde{X}_{i}(k) B_{2, i}+C_{2, i} \tilde{X}_{i+1}(k) D_{2, i}\right) B_{2, i}^{T} \\
& +C_{2, i}^{T}\left(A_{2, i} \widetilde{X}_{i}(k) B_{2, i}+C_{2, i} \widetilde{X}_{i+1}(k) D_{2, i}\right) D_{2, i}^{T} \|^{2} \\
& \leq\left\|\tilde{X}_{i}(k)\right\|^{2}-2 \delta\left(\left\|A_{1, i} \tilde{X}_{i}(k) B_{1, i}+C_{1, i} \tilde{X}_{i+1}(k) D_{1, i}\right\|^{2}\right. \\
& \left.+\left\|A_{2, i} \tilde{X}_{i}(k) B_{2, i}+C_{2, i} \tilde{X}_{i+1}(k) D_{2, i}\right\|^{2}\right) \\
& +\delta^{2}\left(\left\|A_{1, i} B_{1, i}\right\|^{2}+\left\|C_{1, i} D_{1, i}\right\|^{2}+\left\|A_{2, i} B_{2, i}\right\|^{2}+\left\|C_{2, i} D_{2, i}\right\|^{2}\right) \\
& \times\left(\left\|A_{1, i} \tilde{X}_{i}(k) B_{1, i}+C_{1, i} \tilde{X}_{i+1}(k) D_{1, i}\right\|^{2}+\left\|A_{2, i} \tilde{X}_{i}(k) B_{2, i}+C_{2, i} \tilde{X}_{i+1}(k) D_{2, i}\right\|^{2}\right) .
\end{aligned}
$$


By defining the nonnegative definite function $Z(k)$ as follows

$$
Z(k)=\sum_{i=1}^{\theta}\left\|\tilde{X}_{i}(k)\right\|^{2}
$$

we have

$$
\begin{aligned}
& Z(k+1)=\sum_{i=1}^{\theta}\left\|\widetilde{X}_{i}(k+1)\right\|^{2} \\
& \leq \sum_{i=1}^{\theta}\left\|\widetilde{X}_{i}(k)\right\|^{2}-2 \delta \sum_{i=1}^{\theta}\left(\left\|A_{1, i} \tilde{X}_{i}(k) B_{1, i}+C_{1, i} \tilde{X}_{i+1}(k) D_{1, i}\right\|^{2}\right. \\
& \left.+\left\|A_{2, i} \tilde{X}_{i}(k) B_{2, i}+C_{2, i} \tilde{X}_{i+1}(k) D_{2, i}\right\|^{2}\right) \\
& +\delta^{2} \sum_{i=1}^{\theta}\left(\left\|A_{1, i} B_{1, i}\right\|^{2}+\left\|C_{1, i} D_{1, i}\right\|^{2}+\left\|A_{2, i} B_{2, i}\right\|^{2}+\left\|C_{2, i} D_{2, i}\right\|^{2}\right) \\
& \times \sum_{i=1}^{\theta}\left(\left\|A_{1, i} \widetilde{X}_{i}(k) B_{1, i}+C_{1, i} \widetilde{X}_{i+1}(k) D_{1, i}\right\|^{2}\right. \\
& \left.+\left\|A_{2, i} \widetilde{X}_{i}(k) B_{2, i}+C_{2, i} \tilde{X}_{i+1}(k) D_{2, i}\right\|^{2}\right) \\
& \leq Z(0)-\delta\left[2-\delta \sum_{i=1}^{\theta}\left(\left\|A_{1, i} B_{1, i}\right\|^{2}+\left\|C_{1, i} D_{1, i}\right\|^{2}+\left\|A_{2, i} B_{2, i}\right\|^{2}+\left\|C_{2, i} D_{2, i}\right\|^{2}\right)\right] \\
& \times \sum_{r=1}^{k} \sum_{i=1}^{\theta}\left(\left\|A_{1, i} \widetilde{X}_{i}(r) B_{1, i}+C_{1, i} \widetilde{X}_{i+1}(r) D_{1, i}\right\|^{2}\right. \\
& \left.+\left\|A_{2, i} \tilde{X}_{i}(r) B_{2, i}+C_{2, i} \tilde{X}_{i+1}(r) D_{2, i}\right\|^{2}\right) .
\end{aligned}
$$

If the convergence factor $\delta$ is chosen to satisfy in (2.4) then we can conclude that

$$
\begin{aligned}
& \sum_{r=1}^{\infty} \sum_{i=1}^{\theta}\left(\left\|A_{1, i} \tilde{X}_{i}(r) B_{1, i}+C_{1, i} \widetilde{X}_{i+1}(r) D_{1, i}\right\|^{2}\right. \\
& \left.+\left\|A_{2, i} \widetilde{X}_{i}(r) B_{2, i}+C_{2, i} \widetilde{X}_{i+1}(r) D_{2, i}\right\|^{2}\right)<\infty .
\end{aligned}
$$

It follows from the necessary condition of the above series convergence that

$$
\begin{aligned}
& \lim _{r \rightarrow \infty} \sum_{i=1}^{\theta}\left(\left\|A_{1, i} \widetilde{X}_{i}(r) B_{1, i}+C_{1, i} \widetilde{X}_{i+1}(r) D_{1, i}\right\|^{2}\right. \\
& \left.+\left\|A_{2, i} \tilde{X}_{i}(r) B_{2, i}+C_{2, i} \tilde{X}_{i+1}(r) D_{2, i}\right\|^{2}\right)=0 .
\end{aligned}
$$


Hence we deduce that

$$
\lim _{r \rightarrow \infty}\left(A_{1, i} \tilde{X}_{i}(r) B_{1, i}+C_{1, i} \widetilde{X}_{i+1}(r) D_{1, i}\right)=0, \quad \text { for } i=1,2, \ldots, \theta
$$

and

$$
\lim _{r \rightarrow \infty}\left(A_{2, i} \widetilde{X}_{i}(r) B_{2, i}+C_{2, i} \widetilde{X}_{i+1}(r) D_{2, i}\right)=0, \quad \text { for } \quad i=1,2, \ldots, \theta .
$$

Now according to Lemma 1, it can be obtained that

$$
\lim _{r \rightarrow \infty} \tilde{X}_{i}(r)=0, \quad \text { for } i=1,2, \ldots, \theta .
$$

This finishes the proof of Theorem 1.

\section{$3 \quad$ Numerical examples}

In this section, two numerical examples are proposed for the validation of the proposed method. We performed our computations using Matlab software on a Pentium IV.

Example 1. We consider the discrete-time periodic matrix equations

$$
A_{i} X_{i}+X_{i+1} B_{i}=C_{i}, \quad i=1,2,3
$$

over the generalized reflexive matrices $X_{1}, X_{2}, X_{3} \in \mathbb{R}_{r}^{5 \times 5}(P, Q)$ where

$$
\begin{gathered}
A_{1}=\left(\begin{array}{ccccc}
2.6756 & 0.3840 & 0.6085 & 0.0576 & 0.0841 \\
0 & 2.4508 & 0.0158 & 0.3676 & 0.4544 \\
0 & 0 & 2.2324 & 0.6315 & 0.4418 \\
0 & 0 & 0 & 2.0784 & 0.3533 \\
0 & 0 & 0 & 0 & 2.9943
\end{array}\right), \\
A_{2}=\left(\begin{array}{cccccc}
-3.2475 & 0.5915 & 0 & 0 & 0 \\
0.3400 & -3.7362 & 0.2644 & 0 & 0 \\
0.3142 & 0.0381 & -3.2519 & 0.6649 & 0 \\
0.3651 & 0.4586 & 0.8729 & -2.7797 & 0.8903 \\
0.3932 & 0.8699 & 0.2379 & 0.0099 & -2.9985
\end{array}\right), \\
-7.4617 \\
A_{3}=\left(\begin{array}{cccccc}
0.9200 & 0.1939 & 0.5488 & 0.6273 \\
0.0099 & -6.6666 & 0.9048 & 0.9316 & 0.6991 \\
0.7199 & 0.3678 & -7.2374 & 0.3352 & 0.3972 \\
0.7939 & 0.7313 & 0.2344 & 0.3919 & -6.4036
\end{array}\right), \\
B_{1}=\left(\begin{array}{llllll}
9.1529 & 0.7621 & 0.6154 & 0.4057 & 0.0579 \\
0.2311 & 9.2033 & 0.7919 & 0.9355 & 0.3529 \\
0.6068 & 0.0185 & 9.4470 & 0.9169 & 0.8132 \\
0.4860 & 0.8214 & 0.7382 & 8.7898 & 0.0099 \\
0.8913 & 0.4447 & 0.1763 & 0.8936 & 8.3285
\end{array}\right),
\end{gathered}
$$




$$
\begin{aligned}
& B_{2}=\left(\begin{array}{ccccc}
8.8962 & 0.6979 & 0 & 0 & 0 \\
0.6822 & 9.3352 & 0.8998 & 0 & 0 \\
0.3028 & 0.8600 & 9.0740 & 0.2897 & 0 \\
0.5417 & 0.8537 & 0.6449 & 8.5403 & 0.5681 \\
0.1509 & 0.5936 & 0.8180 & 0.5341 & 9.3587
\end{array}\right) \\
& B_{3}=\left(\begin{array}{ccccc}
8.5536 & 0.2259 & 0 & 0 & 0 \\
0.4235 & 8.2233 & 0.3798 & 0 & 0 \\
0.5155 & 0.7604 & 8.0513 & 0.0592 & 0 \\
0.3340 & 0.5298 & 0.6808 & 8.2317 & 0.0150 \\
0.4329 & 0.6405 & 0.4611 & 0.0503 & 8.3431
\end{array}\right) \\
& C_{1}=\left(\begin{array}{ccccc}
4.7707 & 11.0062 & 2.2473 & 14.7423 & 37.2686 \\
40.4523 & 3.4924 & 15.8879 & 3.2050 & 1.6293 \\
3.1070 & 19.8112 & 3.2951 & 23.4979 & 16.1410 \\
10.5080 & 1.1985 & 7.9500 & 1.4258 & 0.9173 \\
3.0847 & 28.6604 & 3.8242 & 26.0354 & 17.0594
\end{array}\right), \\
& C_{2}=\left(\begin{array}{ccccc}
5.4803 & 17.7438 & 9.5312 & 17.6358 & 59.4288 \\
54.2694 & 8.1642 & 18.1172 & 1.5983 & 1.7068 \\
5.7146 & 30.8417 & 8.6997 & 30.0182 & 28.3407 \\
17.0218 & 6.4641 & 11.3355 & 4.4598 & 4.2447 \\
9.4985 & 44.6355 & 10.9847 & 33.1244 & 30.2029
\end{array}\right), \\
& C_{3}=\left(\begin{array}{ccccc}
9.2292 & -2.9557 & 4.4280 & -7.0361 & -37.8309 \\
-32.3081 & 7.3022 & -10.3963 & 6.7477 & 5.2847 \\
4.4957 & -13.3424 & 2.7645 & -18.3820 & -12.0716 \\
-3.6924 & 5.6008 & -4.7002 & 5.8530 & 9.0744 \\
7.5784 & -18.1886 & 3.9682 & -16.2704 & -7.4623
\end{array}\right)
\end{aligned}
$$

and

$$
P=\left(\begin{array}{ccccc}
1 & 0 & 0 & 0 & 0 \\
0 & -1 & 0 & 0 & 0 \\
0 & 0 & 1 & 0 & 0 \\
0 & 0 & 0 & -1 & 0 \\
0 & 0 & 0 & 0 & 1
\end{array}\right), Q=\left(\begin{array}{ccccc}
-1 & 0 & 0 & 0 & 0 \\
0 & 1 & 0 & 0 & 0 \\
0 & 0 & -1 & 0 & 0 \\
0 & 0 & 0 & 1 & 0 \\
0 & 0 & 0 & 0 & 1
\end{array}\right) .
$$

By applying Algorithm 1 with the initial generalized reflexive matrices $X_{1}(1)$ $=X_{2}(1)=X_{3}(1)=0$ and several values of parameter $\delta$, we obtain results presented in Figure 1 where

$$
r(k)=\log _{10}\left(\sqrt{\sum_{i=1}^{3}\left\|C_{i}-A_{i} X_{i}(k)-X_{i+1}(k) B_{i}\right\|^{2}}\right) .
$$

After 125 iterations, we obtain the generalized reflexive solutions of the discrete time periodic matrix equations as follows:

$$
X_{1}^{*}=\left(\begin{array}{ccccc}
0 & 0.3757 & 0 & 0.4919 & 1.8847 \\
1.9034 & 0 & 0.6286 & 0 & 0 \\
0 & 0.8185 & 0 & 1.0121 & 0.8036 \\
0.4947 & 0 & 0.3500 & 0 & 0 \\
0 & 1.2219 & 0 & 1.0828 & 0.8231
\end{array}\right) \in \mathbb{R}_{r}^{5 \times 5}(P, Q),
$$




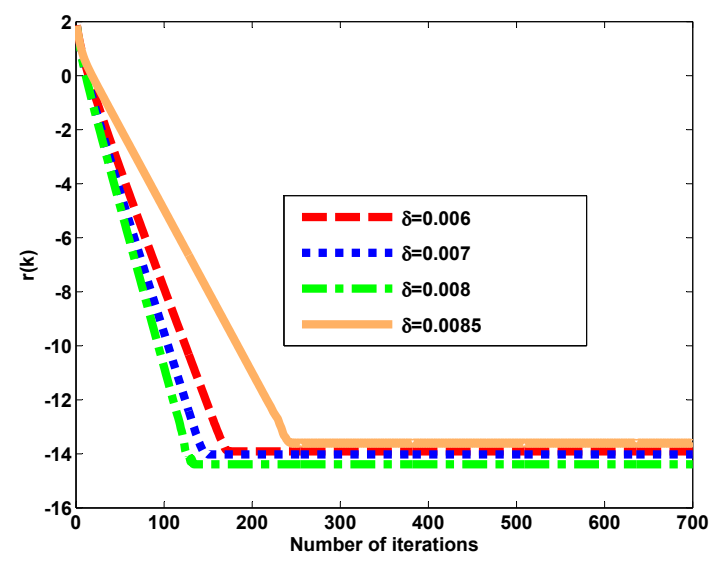

Figure 1. The residuals for Example 1

$$
\begin{aligned}
& X_{2}^{*}=\left(\begin{array}{ccccc}
0 & 0.7514 & 0 & 0.9838 & 3.7693 \\
3.8068 & 0 & 1.2571 & 0 & 0 \\
0 & 1.6371 & 0 & 2.0242 & 1.6072 \\
0.9893 & 0 & 0.7001 & 0 & 0 \\
0 & 2.4438 & 0 & 2.1657 & 1.6463
\end{array}\right) \in \mathbb{R}_{r}^{5 \times 5}(P, Q), \\
& X_{3}^{*}=\left(\begin{array}{ccccc}
0 & 1.5029 & 0 & 1.9677 & 7.5386 \\
7.6135 & 0 & 2.5142 & 0 & 0 \\
0 & 3.2742 & 0 & 4.0484 & 3.2144 \\
1.9786 & 0 & 1.4002 & 0 & 0 \\
0 & 4.8875 & 0 & 4.3314 & 3.2925
\end{array}\right) \in \mathbb{R}_{r}^{5 \times 5}(P, Q) .
\end{aligned}
$$

The results show that Algorithm 1 can quickly obtain the solutions of the discrete-time periodic matrix equations.

Example 2. We study the coupled discrete-time periodic matrix equations

$$
\left\{\begin{array}{l}
X_{i}+A_{i} X_{i+1} B_{i}=C_{i}, \\
D_{i} X_{i} E_{i}+X_{i+1}=F_{i},
\end{array} \quad i=1,2,3\right.
$$

with the following parameters

$$
\begin{aligned}
A_{1} & =\left(\begin{array}{ccccc}
8.6756 & 0.3840 & 0.6085 & 0.0576 & 0.0841 \\
0 & 8.4508 & 0.0158 & 0.3676 & 0.4544 \\
0 & 0 & 8.2324 & 0.6315 & 0.4418 \\
0 & 0 & 0 & 8.0784 & 0.3533 \\
0 & 0 & 0 & 0 & 8.9943
\end{array}\right), \\
A_{2} & =\left(\begin{array}{llllll}
7.1271 & 0.5915 & 0 & 0 & 0 \\
0.3400 & 6.9757 & 0.2644 & 0 & 0 \\
0.3142 & 0.0381 & 6.5725 & 0.6649 & 0 \\
0.3651 & 0.4586 & 0.8729 & 7.5205 & 0.8903 \\
0.3932 & 0.8699 & 0.2379 & 0.0099 & 7.4683
\end{array}\right),
\end{aligned}
$$




$$
\begin{aligned}
& A_{3}=\left(\begin{array}{ccccc}
-5.4617 & 0.9200 & 0.1939 & 0.5488 & 0.6273 \\
0.0099 & -4.6666 & 0.9048 & 0.9316 & 0.6991 \\
0.4199 & 0.3678 & -5.2374 & 0.3352 & 0.3972 \\
0.7537 & 0.6208 & 0.6318 & -4.4845 & 0.4136 \\
0.7939 & 0.7313 & 0.2344 & 0.3919 & -4.4036
\end{array}\right) \\
& B_{1}=\left(\begin{array}{ccccc}
3.2526 & -0.7621 & -0.6154 & -0.4057 & -0.0579 \\
-0.2311 & 4.2903 & -0.7919 & -0.9355 & -0.3529 \\
-0.6068 & -0.0185 & 3.6033 & -0.9169 & -0.8132 \\
-0.4860 & -0.8214 & -0.7382 & 3.9692 & -0.0099 \\
-0.8913 & -0.4447 & -0.1763 & -0.8936 & 4.0508
\end{array}\right) \text {, } \\
& B_{2}=\left(\begin{array}{ccccc}
9.7027 & 0.6979 & 0.4966 & 0.6602 & 0.7271 \\
0 & 9.9568 & 0.8998 & 0.3420 & 0.3093 \\
0 & 0 & 9.2523 & 0.2897 & 0.8385 \\
0 & 0 & 0 & 9.1991 & 0.5681 \\
0 & 0 & 0 & 0 & 9.9883
\end{array}\right) \\
& B_{3}=\left(\begin{array}{ccccc}
8.5536 & 0.2259 & 0 & 0 & 0 \\
0.4235 & 8.2233 & 0.3798 & 0 & 0 \\
0.5155 & 0.7604 & 8.0513 & 0.0592 & 0 \\
0.3340 & 0.5298 & 0.6808 & 8.2317 & 0.0150 \\
0.4329 & 0.6405 & 0.4611 & 0.0503 & 8.3431
\end{array}\right) \text {, } \\
& D_{1}=\left(\begin{array}{ccccc}
3.9317 & -0.5711 & -0.4319 & -0.9159 & -0.7327 \\
-0.5485 & 3.7974 & -0.6343 & -0.6020 & -0.4222 \\
-0.2618 & -0.9623 & 3.9515 & -0.2536 & -0.9614 \\
-0.5973 & -0.7505 & -0.0839 & 3.7528 & -0.0721 \\
-0.0493 & -0.7400 & -0.9455 & -0.5134 & 3.9206
\end{array}\right) \text {, } \\
& D_{2}=\left(\begin{array}{ccccc}
3.6206 & 0.1614 & 0.8121 & 0.3756 & 0.9566 \\
0 & 3.4906 & 0.6101 & 0.1662 & 0.1472 \\
0 & 0 & 3.6084 & 0.8332 & 0.8699 \\
0 & 0 & 0 & 3.4648 & 0.7694 \\
0 & 0 & 0 & 0 & 3.4116
\end{array}\right) \\
& D_{3}=\left(\begin{array}{ccccc}
-7.2833 & 0.1122 & 0 & 0 & 0 \\
0.3941 & -6.8592 & 0.2816 & 0 & 0 \\
0.5030 & 0.4668 & -7.3344 & 0.9028 & 0 \\
0.7220 & 0.0147 & 0.7085 & -7.4818 & 0.5208 \\
0.3062 & 0.6641 & 0.7839 & 0.8045 & -6.6376
\end{array}\right) \text {, } \\
& E_{1}=\left(\begin{array}{ccccc}
10.1171 & -0.9327 & -0.2093 & -0.3193 & -0.1998 \\
0 & 10.9492 & -0.4551 & -0.3749 & -0.0495 \\
0 & 0 & 10.9331 & -0.8678 & -0.5667 \\
0 & 0 & 0 & 10.2905 & -0.1219 \\
0 & 0 & 0 & 0 & 10.0954
\end{array}\right) \text {, } \\
& E_{2}=\left(\begin{array}{ccccc}
-3.0278 & -0.8194 & 0 & 0 & 0 \\
-0.2882 & -3.6955 & -0.7536 & 0 & 0 \\
-0.8167 & -0.5602 & -4.3628 & -0.1834 & 0 \\
-0.9855 & -0.2440 & -0.2141 & -3.9130 & -0.6773 \\
-0.0174 & -0.8220 & -0.6021 & -0.1703 & -4.8245
\end{array}\right) \text {, }
\end{aligned}
$$




$$
\begin{aligned}
& E_{3}=\left(\begin{array}{ccccc}
-10.8912 & 0 & 0 & 0 & 0 \\
0 & -10.5019 & 0 & 0 & 0 \\
0 & 0 & -10.1112 & 0 & 0 \\
0 & 0 & 0 & -10.5195 & 0 \\
0 & 0 & 0 & 0 & -10.5216
\end{array}\right) \text {, } \\
& C_{1}=10^{3}\left(\begin{array}{ccccc}
-0.2241 & -0.2708 & 0.2276 & 1.0767 & 0.0841 \\
0.9796 & 0.0200 & -0.2286 & -0.1756 & -0.0375 \\
-0.2072 & -0.0106 & 0.8505 & -0.0594 & 0.1004 \\
0.3911 & 0.8389 & -0.2531 & -0.2562 & -0.0840 \\
0.4749 & 0.4448 & -0.1961 & -0.1827 & -0.0544
\end{array}\right) \text {, } \\
& C_{2}=10^{3}\left(\begin{array}{lllll}
0.3894 & 0.0856 & 1.7398 & 4.7341 & 1.1657 \\
4.6264 & 1.0147 & 0.5306 & 0.6037 & 0.4854 \\
0.2283 & 0.3661 & 3.9528 & 1.3614 & 1.7330 \\
2.9085 & 4.4464 & 1.1209 & 0.7294 & 0.6241 \\
3.0508 & 2.4571 & 0.5878 & 0.5811 & 0.4184
\end{array}\right) \\
& C_{3}=\left(\begin{array}{ccccc}
168.0550 & 37.2777 & -298.8015 & -717.0216 & -94.5945 \\
-482.8643 & 58.0143 & 121.2561 & 33.3031 & 35.8865 \\
52.3395 & -13.3380 & -592.5257 & -106.8510 & -179.0708 \\
-158.3376 & -374.7244 & 111.1639 & 131.8542 & 40.3635 \\
-152.6549 & -162.7658 & 72.9395 & 123.7053 & 25.5157
\end{array}\right) \text {, } \\
& F_{1}=\left(\begin{array}{ccccc}
-233.8164 & -182.3232 & 229.7091 & 727.3289 & 67.2928 \\
601.4525 & -65.0355 & -158.1209 & -132.0087 & -45.6560 \\
-267.8252 & -112.8876 & 699.5537 & 90.0978 & 161.7304 \\
179.6600 & 522.0632 & -81.1046 & -129.9805 & -19.9160 \\
185.4702 & 213.6419 & -177.5114 & -48.7498 & -41.4132
\end{array}\right) \text {, } \\
& F_{2}=\left(\begin{array}{ccccc}
-282.2199 & -206.4360 & -351.0661 & -472.2401 & -202.9035 \\
-333.6208 & -195.0378 & -105.5166 & -24.2335 & -31.2039 \\
-216.4398 & -247.9265 & -482.4659 & -126.7748 & -165.6702 \\
-202.4713 & -378.4970 & -76.5490 & 0 & 0 \\
-155.3920 & -199.6648 & -36.9034 & 0 & 0
\end{array}\right) \text {, } \\
& F_{3}=10^{3}\left(\begin{array}{ccccc}
-0.0829 & -0.0115 & 1.9069 & 5.4131 & 0.7457 \\
5.0859 & 0.7071 & -0.2809 & -0.3410 & -0.0962 \\
-0.6546 & -0.5434 & 4.5224 & 0.9083 & 1.4102 \\
2.3706 & 3.9606 & -0.6364 & -0.6582 & -0.2144 \\
1.6942 & 1.4985 & -0.5756 & -0.3633 & -0.1869
\end{array}\right)
\end{aligned}
$$

and

$$
P=\left(\begin{array}{ccccc}
1 & 0 & 0 & 0 & 0 \\
0 & -1 & 0 & 0 & 0 \\
0 & 0 & 1 & 0 & 0 \\
0 & 0 & 0 & -1 & 0 \\
0 & 0 & 0 & 0 & -1
\end{array}\right), Q=\left(\begin{array}{ccccc}
-1 & 0 & 0 & 0 & 0 \\
0 & -1 & 0 & 0 & 0 \\
0 & 0 & 1 & 0 & 0 \\
0 & 0 & 0 & 1 & 0 \\
0 & 0 & 0 & 0 & 1
\end{array}\right)
$$

We apply Algorithm 1 with the initial matrices $X_{1}(1)=X_{2}(1)=X_{3}(1)=0$ and several values of parameter $\delta$ to solve the coupled discrete-time periodic 
matrix equations. Figure 2 shows the performance of Algorithm 1 with the residuals

$$
r(k)=\log _{10}\left(\sqrt{\sum_{i=1}^{3}\left[\left\|C_{i}-X_{i}(k)-A_{i} X_{i+1}(k) B_{i}\right\|^{2}+\left\|F_{i}-D_{i} X_{i}(k) E_{i}-X_{i+1}(k)\right\|^{2}\right]}\right) .
$$

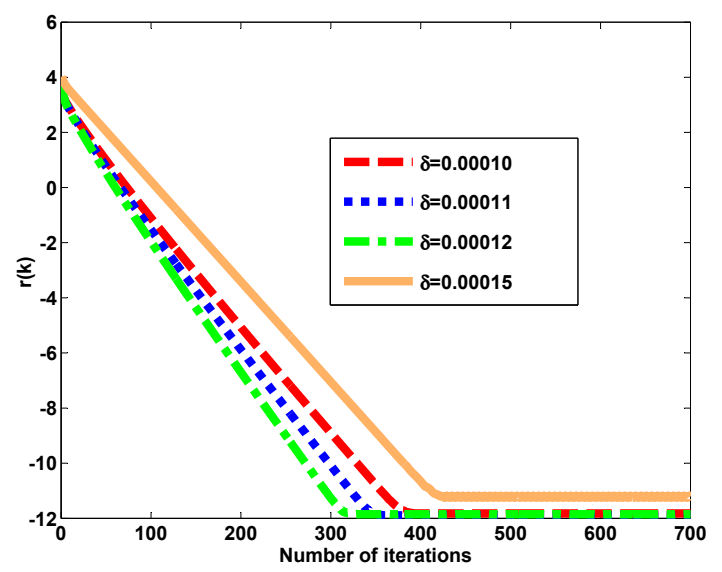

Figure 2. The residuals for Example 2

After 315 iterations, Algorithm 1 can compute the generalized reflexive solutions of the coupled discrete-time periodic matrix equations by the following:

$$
\begin{aligned}
& X_{1}^{*}=\left(\begin{array}{ccccc}
0 & 0 & 6.4515 & 17.6055 & 2.4249 \\
16.9630 & 2.4456 & 0 & 0 & 0 \\
0 & 0 & 15.6354 & 4.1373 & 4.7196 \\
7.8725 & 13.0642 & 0 & 0 & 0 \\
8.4808 & 7.1767 & 0 & 0 & 0
\end{array}\right) \in \mathbb{R}_{r}^{5 \times 5}(P, Q) \\
& X_{2}^{*}=\left(\begin{array}{ccccc}
0 & 0 & 12.9031 & 35.2109 & 4.8498 \\
33.9259 & 4.8911 & 0 & 0 & 0 \\
0 & 0 & 31.2709 & 8.2746 & 9.4392 \\
15.7451 & 26.1284 & 0 & 0 & 0 \\
16.9616 & 14.3533 & 0 & 0 & 0
\end{array}\right) \in \mathbb{R}_{r}^{5 \times 5}(P, Q) \\
& X_{3}^{*}=\left(\begin{array}{ccccc}
0 & 0 & 25.8061 & 70.4219 & 9.6997 \\
67.8519 & 9.7822 & 0 & 0 & 0 \\
0 & 0 & 62.5417 & 16.5491 & 18.8784 \\
31.4902 & 52.2568 & 0 & 0 & 0 \\
33.9232 & 28.7066 & 0 & 0 & 0
\end{array}\right) \in \mathbb{R}_{r}^{5 \times 5}(P, Q) .
\end{aligned}
$$

From Figure 2, we can see that Algorithm 1 is effective to solve discrete-time periodic matrix equations. 


\section{Conclusions}

In this paper, the generalized reflexive solutions of general coupled discretetime periodic matrix equations (1.4) were studied. We proposed a gradient based iterative method to solve (1.4) over the generalized reflexive matrices. It was proven that the iterative solution converges to the generalized reflexive solutions for any initial generalized reflexive matrices. The numerical examples demonstrated the potential of this method in solving (1.4) over the generalized reflexive matrices.

\section{Acknowledgements}

The author is deeply indebted to four anonymous referees for providing useful comments and constructive suggestion.

\section{References}

[1] P. Benner, M.S. Hossain and T. Stykel. Low-rank iterative solution of periodic projected Lyapunov equations and their application in model reduction of periodic descriptor systems. Chemnitz Scientific Computing; CSC/11-01; 2011. ISBN/ISSN: 1864-0087. Chemnitz University of Technology; Germany, 2011.

[2] P. Benner, M.S. Hossain and T. Stykel. Model reduction of periodic descriptor systems using balanced truncation. In M. Hinze P. Benner and J. ter Maten(Eds.), Model Reduction in Circuit Simulation, volume 74 of Lecture Notes in Electrical Engineering, pp. 193-206, Springer-Verlag, Berlin, Heidelberg, 2011.

[3] S. Bittanti and P. Colaneri. Analysis of discrete-time linear periodic systems. Control and Dynamics Systems, 78:313-339, 1996. http://dx.doi.org/10.1016/S0090-5267(96)80056-9.

[4] R. Byers and N. Rhee. Cyclic Schur and Hessenberg Schur numerical methods for solving periodic Lyapunov and Sylvester equations. Technical Report, Dept. of Mathematics, Univ. of Missouri at Kansas City, 1995.

[5] H.C. Chen. Generalized reflexive matrices: special properties and applications. SIAM Journal on Matrix Analysis and Applications, 19(1):140-153, 1998. http://dx.doi.org/10.1137/S0895479895288759.

[6] E.K.W. Chu, H.Y. Fan and W.W. Lin. Projected generalized discrete-time periodic Lyapunov equations and balanced realization of periodic descriptor systems. SIAM Journal on Matrix Analysis and Applications, 29(3):982-1006, 2007. http://dx.doi.org/10.1137/040606715.

[7] M. Dehghan and M. Hajarian. An iterative algorithm for the reflexive solutions of the generalized coupled Sylvester matrix equations and its optimal approximation. Applied Mathematics and Computation, 202(2):571-588, 2008. http://dx.doi.org/10.1016/j.amc.2008.02.035.

[8] M. Dehghan and M. Hajarian. The general coupled matrix equations over generalized bisymmetric matrices. Linear Algebra and its Applications, 432(6):15311552, 2010. http://dx.doi.org/10.1016/j.laa.2009.11.014. 
[9] M. Dehghan and M. Hajarian. An iterative method for solving the generalized coupled Sylvester matrix equations over generalized bisymmetric matrices. Applied Mathematical Modelling, 34(3):639-654, 2010. http://dx.doi.org/10.1016/j.apm.2009.06.018.

[10] M. Dehghan and M. Hajarian. Analysis of an iterative algorithm to solve the generalized coupled Sylvester matrix equations. Applied Mathematical Modelling, 35(7):3285-3300, 2011. http://dx.doi.org/10.1016/j.apm.2011.01.022.

[11] F. Ding and T. Chen. Gradient based iterative algorithms for solving a class of matrix equations. IEEE Transactions on Automatic Control, 50(8):1216-1221, 2005. http://dx.doi.org/10.1109/TAC.2005.852558.

[12] F. Ding and T. Chen. Iterative least squares solutions of coupled Sylvester matrix equations. Systems \& Control Letters, 54(2):95-107, 2005. http://dx.doi.org/10.1016/j.sysconle.2004.06.008.

[13] F. Ding and T. Chen. On iterative solutions of general coupled matrix equations. SIAM Journal on Control and Optimization, 44(6):2269-2284, 2006. http://dx.doi.org/10.1137/S0363012904441350.

[14] F. Ding, P.X. Liu and J. Ding. Iterative solutions of the generalized Sylvester matrix equations by using the hierarchical identification principle. Applied Mathematics and Computation, 197(1):41-50, 2008. http://dx.doi.org/10.1016/j.amc.2007.07.040.

[15] F. Ding and H. Zhang. Gradient-based iterative algorithm for a class of the coupled matrix equations related to control systems. IET Control Theory $\mathcal{E}$ Applications, 8(15):1588-1595, 2014. http://dx.doi.org/10.1049/iet-cta.2013.1044.

[16] J. Ding, Y. Liu and F. Ding. Iterative solutions to matrix equations of the form $A_{i} X B_{i}=F_{i}$. Computers \& Mathematics with Applications, 59(11):3500-3507, 2010. http://dx.doi.org/10.1016/j.camwa.2010.03.041.

[17] R. Granat, I. Jonsson and B. Kàgström. Recursive blocked algorithms for solving periodic triangular Sylvester-type matrix equations. In Proc. of the 8th international conference on Applied parallel computing: state of the art in scientific computing, pp. 531-539. Springer, 2006.

[18] M. Hajarian. Developing the CGLS algorithm for the least squares solutions of the general coupled matrix equations. Mathematical Methods in the Applied Sciences, 37(17):2782-2798, 2014. http://dx.doi.org/10.1002/mma.3017.

[19] M.S. Hossain and P. Benner. Iterative solvers for periodic matrix equations and model reduction for periodic control systems. In 7 th International Conference on Electrical and Computer Engineering 20-22, Dhaka, Bangladesh, 2012.

[20] D. Kressner. Large periodic Lyapunov equations: Algorithms and applications. In Proc. of ECC03, Cambridge, UK, 2003.

[21] Q. Niu, X. Wang and L.-Z. Lu. A relaxed gradient based algorithm for solving Sylvester equations. Asian Journal of Control, 13(3):461-464, 2011. http://dx.doi.org/10.1002/asjc.328.

$[22]$ Z. Peng. The $(R, S)$-symmetric least squares solutions of the general coupled matrix equations. Linear and Multilinear Algebra, 63(6):1086-1105, 2015. http://dx.doi.org/10.1080/03081087.2014.918615.

[23] D.K. Salkuyeh and F.P.A. Beik. Minimum norm least-squares solution to general complex coupled linear matrix equations via iteration. Filomat, 29(6):1389-1407, 2015. http://dx.doi.org/10.2298/FIL1506389S. 
[24] R. Smith. Matrix equation $X A+B X=C$. SIAM Journal on Applied Mathematics, 16(1):198-201, 1968. http://dx.doi.org/10.1137/0116017.

[25] J. Sreedhar and P. Van Dooren. Periodic Schur form and some matrix equations. In Proc. of the Symposium on the Mathematical Theory of Networks and Systems (MTNS'93), pp. 339-362, Regensburg, Germany, 1994.

[26] T. Stykel. On some norms for descriptor systems. IEEE Transactions on Automatic Control, 51(5):842-847, 2006. http://dx.doi.org/10.1109/TAC.2006.875010.

[27] T. Stykel. Low-rank iterative methods for projected generalized Lyapunov equations. Electronic Transactions on Numerical Analysis, 30:187-202, 2008.

[28] A. Varga. Periodic Lyapunov equations: some applications and new algorithms. International Journal of Control, 67(1):69-87, 1997. http://dx.doi.org/10.1080/002071797224360.

[29] A. Varga. On computing minimal realizations of periodic descriptor systems. In Proc. of IFAC Workshop on Periodic Control Systems, pp. 175-180, Anichkov Palace, Russia, 2007.

[30] X. Wang, L. Dai and D. Liao. A modified gradient based algorithm for solving Sylvester equations. Applied Mathematics and Computation, 218(9):5620-5628, 2012. http://dx.doi.org/10.1016/j.amc.2011.11.055.

[31] X. Wang and D. Liao. The optimal convergence factor of the gradient based iterative algorithm for linear matrix equations. Filomat, 26(3):607-613, 2012. http://dx.doi.org/10.2298/FIL1203607W.

[32] H. Zhang and J. Wang. No $\mathrm{N}_{\mathrm{x}}$ sensor ammonia-cross-sensitivity factor estimation in diesel engine selective catalytic reduction systems. In International Conference on Mechatronics and Control (ICMC), pp. 137(6), 061015 (Jun 01, 2015) (9 pages), 2014.

[33] H. Zhang and J. Wang. Robust two-mode-dependent controller design for networked control systems with random delays modelled by Markov chains. International Journal of Control, 88(12):2499-2509, 2015. http://dx.doi.org/10.1080/00207179.2015.1048293.

[34] B. Zhou, G.R. Duan and Z.Y. Li. Gradient based iterative algorithm for solving coupled matrix equations. Systems $\&$ Control Letters, 58(5):327-333, 2009. http://dx.doi.org/10.1016/j.sysconle.2008.12.004.

[35] B. Zhou, G.R. Duan and Z. Lin. A parametric periodic Lyapunov equation with application in semi-global stabilization of discrete-time periodic systems subject to actuator saturation. Automatica, 47(2):316-325, 2011. http://dx.doi.org/10.1016/j.automatica.2010.10.011. 